\title{
Cartografías conectivas: un acercamiento a la construcción de redes sociodigitales del movimiento \#LGBT
}

Connective cartographies: an approach to the construction of socio-digital networks of the \#LGBT movement

Cartografias conectivas: uma abordagem à construção de redes sócio-digitais do movimento \#LGBT

\author{
Raul Anthony OLMEDO NERI \\ Universidad Tecnológica de México - UNITEC MÉXICO - Campus en Línea \\ México \\ raulanthonyn@yahoo.com.mx
}

Chasqui. Revista Latinoamericana de Comunicación

$N .^{o}$ 147, agosto-noviembre 2021 (Sección Monográfico, pp. 121-140)

ISSN 1390-1079 / e-ISSN 1390-924X

Ecuador: CIESPAL

Recibido: 20-03-2021 / Aprobado: 10-07-2021 


\title{
Resumen
}

Eltrabajo analiza la red sociodigitalinternacional generada alrededor del hashtag \#LGBT. El marco teórico descansa en la perspectiva de la red como paradigma para abordar la apropiación de Internet por parte de los movimientos sociales. Se utiliza el método de Análisis de Redes Sociales (ARS) a una base de datos obtenida mediante minería de datos con el programa NodeXL y visualizando las redes mediante Gephi. Entre los resultados obtenidos resalta que la red se constituye por comunidades estrechamente vinculadas entre sí y en menor medida con otras. Además, la relevancia de los nodos cambia de acuerdo con la cantidad de vínculos, su direccionalidad o a su reconocimiento/función para la comunidad LGBT y la propia plataforma (Twitter).

Palabras clave: Twitter, apropiación, redes sociales, movimiento LGBT, ARS.

\begin{abstract}
The work analyzes the international sociodigital network generated around the hashtag \#LGBT. The theoretical framework rests on the perspective of the network as a paradigm to address the appropriation of the Internet by social movements. The Social Network Analysis (ARS) method is used to a database obtained by data mining with the NodeXL program and visualizing the networks using Gephi. Among the results obtained, it stands out that the network is made up of communities closely linked to each other and to a lesser extent with others. In addition, the relevance of the nodes changes according to the number of links, their directionality or their recognition / function for the LGBT community and the platform itself (Twitter).
\end{abstract}

Keywords: Twitter, appropriation, social media, LGBT movement, SNA.

\section{Resumo}

O trabalho analisa a rede sócio-digital internacional gerada em torno da hashtag \#LGBT. O referencial teórico apóia-se na perspectiva da rede como paradigma para abordar a apropriação da Internet pelos movimentos sociais. O método Análise de Redes Sociais (ARS) é utilizado para um banco de dados obtido por mineração de dados com o programa NodeXL e visualização das redes utilizando Gephi. Dentre os resultados obtidos, destaca-se que a rede é composta por comunidades intimamente ligadas entre si e em menor grau com outras. Além disso, a relevância dos nós muda de acordo com o número de links, sua direcionalidade ou seu reconhecimento / função para a comunidade LGBT e para a própria plataforma (Twitter).

Palavras chave: Twitter, apropriação, redes sociais, movimento LGBT, ARS. 


\section{Introducción}

Los dispositivos tecnológicos e Internet se han convertido en un rasgo característico de la sociedad contemporánea. El uso multipropósito que le dan las personas muestra tanto la maleabilidad de este mundo virtual (Diodato, 2011) como la creatividad del individuo del siglo XXI para apropiarlo socialmente y encarnar prácticas híbridas. Dichas prácticas derivan de la cotidianidad hiperconectada (Rodríguez Cano, 2020), la cual difumina la dicotomía online/ offline ante la complejidad de una sociedad onlife (Floridi, 2015).

De todos esos usos posibles, se analiza el campo fértil y en proceso de expansión que los movimientos sociales han creado al replicar y sincronizar sus estrategias mediante el uso de sitios web y plataformas sociodigitales como Facebook y Twitter. Concretamente, se realiza un acercamiento desde el paradigma de la red (Rovira, 2012) a las relaciones de interacción-comunicación (Olmedo Neri, 2020) de la comunidad de la diversidad sexual alrededor del hashtag \#LGBT durante junio de 2020, con la finalidad de comprender desde la comunicación (Mattoni \& Treré, 2014) estas novedosas formas de activismo onlife.

Así, el objetivo de este trabajo pretende identificar la red sociodigital formada alrededor de un hashtag creado por uno de los movimientos sociales contemporáneos más importantes a escala internacional (Martel, 2013), con la finalidad de analizar su estructura y reconocer la posición estratégica que adquieren algunos perfiles dentro de lo que se propone definir como cartografías conectivas.

Para ello, la estructura de la investigación muestra la construcción teórica alrededor de la vinculación Internet-movimientos sociales, para después definir las implicaciones metodológicas del uso del Análisis de Redes Sociales (ARS) dentro del espacio digital y con ello obtener la visualización de estas redes de interacción basadas en los flujos de información que yacen en Twitter. Posteriormente, se hace una presentación y discusión de los resultados, para finalmente delinear conclusiones alrededor de este acercamiento.

\section{Marco teórico}

La red como metáfora, que en sus primeros años solo alcanzaba un nivel abstracto, ha cobrado en la actualidad una materialidad teórica, metodológica y empírica de proporciones tan relevantes que en algunos casos encarna procesos y prácticas de la sociedad contemporánea: sociedad-red (Castells, 2010), movimientos-red (Castells, 2015), capitalismo de redes (Luna, 2004), redes de interacción-comunicación (Olmedo Neri, 2020), teoría del actor-red (Latour, 2008), entre otras, son ejemplos de la proliferación y flexibilidad de este concepto que hoy se sitúa como un paradigma (Kadushin, 2013; Lozares, 2005). 
$\mathrm{Su}$ flexibilidad permite convertirla en un puente para vincular áreas del conocimiento, perspectivas metodológicas y análisis que en conjunto redimensionan un objeto de estudio. Uno de los fenómenos que evidencia esta sinergia se da mediante el uso que les dan los movimientos sociales a las plataformas sociodigitales.

En un primer momento, pensar el activismo onlife desde la noción de red en el ámbito comunicativo implica reconocer dos niveles de estudio: a nivel externo Internet puede entenderse como un nuevo medio de comunicación sociodigital que se inserta y asimila en el ecosistema mediático (Treré, 2020), y que puede ser usado por los propios movimientos sociales; mientras que a nivel interno el espacio digital podrá entenderse como medio y como contexto donde se desarrollan no solo nuevas formas de socialización (Quinn \& Papacharissi, 2018), sino prácticas comunicativas mediadas por el soporte de Internet, la estructura reticular de su funcionamiento y la apropiación digital que haga el individuo.

Para este trabajo resultan relevantes los elementos situados a nivel interno, aunque es importante reconocer que en el nivel externo encuadran indagaciones desde la propia Economía Política de la Comunicación (Fuchs, 2015) y la Ecología de Medios (Scolari, 2015), para entender los conflictos que se desatan con este nuevo medio.

Al enfocar el análisis al nivel interno, es posible decir que tanto la estructura organizativa de movimientos sociales como el LGBT+, feminista, estudiantil y altermundista, así como la propia arquitectura de Internet, descansa en la noción de red. Lo anterior es fundamental para entender parte de sus lógicas de operación, acción y comunicación.

La organización y funcionamiento en red dota a los integrantes de un movimiento social (activistas, organizaciones sociales, aliados y miembros) de una autonomía para desarrollar sus actividades de incidencia y resistencia sin perder su capacidad de organización y trabajo con otros integrantes en momentos de coyuntura. Por su parte, la estructura reticular en Internet permite que este espacio-no-físico (García Calderón \& Olmedo Neri, 2019) adquiera un carácter descentralizado, donde los usuarios participan en la intervención del contenido que transita de forma transmedia y cuyos flujos de información se vuelven ubicuos, redundantes y multidireccionales (Meneses, 2015b).

Así, estos movimientos sociales encuentran en Internet no solo una afinidad estructural, sino también un potencial y permanente espacio/herramienta para incrementar el alcance de sus acciones y estrategias. De allí que el carácter comunicativo de estas acciones colectivas tienda a una mayor visibilidad; no es que antes no la tuvieran, sino que con el contexto actual ya no puede relegarse a nivel secundario: actualmente la dimensión comunicativa en tanto medio (infraestructura) y práctica (comunic-acción) se han vuelto "una característica fundamental de los movimientos contemporáneos” (Candón-Mena, 2019, p. 24). 
Por tanto, recurrir a la noción de red hace factible analizar este proceso de convergencia, de hibridación mediática (Treré, 2020) y apropiación social (Crovi, 2012). De hecho, ya existen estudios de caso que dan cuenta de este proceso de sinergia comunicativa y de acción: sea en el movimiento feminista (Acosta \& Lassi, 2020; Pedraza Bucio \& Rodríguez Cano, 2019), en menor medida sobre la comunidad LGBT+ (Acosta, 2020; Ciszek, 2017; Enguix Grau, 2016; Olmedo Neri, 2019, 2020), estudiantil (Meneses, 2015a; Reguillo, 2013) o altermundista (Castells, 2005; Rheingold, 2004; Reguillo, 2017; Rovira, 2017), en todos estos movimientos sociales existe un expertise que se ha generado a través del ensayo y error sobre el uso de Internet y las plataformas sociodigitales, así como de su replicación mediante el diálogo inter-movimientos y la observación.

Resulta menester anotar que los estudios sobre el movimiento LGBT+ y el uso de Internet dentro de sus estrategias de acción y comunicación son escasos porque la constitución del objeto de estudio se ha centrado en ámbitos más históricos y de orden organizacional (Martínez Carmona, González Villarreal, Mogrovejo Aquise \& Sandoval Rebollo, 2020), por lo que este acercamiento desde las interacciones y el contenido que se crea y difunde en las plataformas sociodigitales abona a esta área de conocimiento en procesos de consolidación.

$\mathrm{Al}$ asumir la red como paradigma es posible reconocer tres tipos de redes que el individuo desarrolla en el espacio virtual: sociales, digitales y sociodigitales. Aunque existe una clara diferenciación entre redes sociales y sociodigitales (Flores-Márquez, 2017) es necesario reconocer un tercer tipo de redes (digitales) dado que presentan características de complementariedad y expansión con las otras dos.

De esta manera, las redes sociales son aquellos vínculos que desarrolla el individuo dentro de los grupos donde se desempeña; estos enlaces están anclados a la biografía del individuo y a los territorios donde construye su identidad. La familia, el trabajo y la escuela son espacios donde las personas construyen vínculos de diversas fuentes, fortaleciéndolos o modificándolos a partir de su devenir histórico. Una característica de estos vínculos es que no pueden ser fácilmente eliminados en el espacio offline, pero sí en el ámbito online. De hecho, existe la posibilidad de replicar estos lazos de manera parcial o total de acuerdo con el interés y el propio autoritarismo que la plataforma sociodigital dota al individuo para decidir si aceptar o no dicha vinculación (Bauman \& Leoncini, 2018).

Las redes digitales, por su parte, aluden a aquellos vínculos que se desarrollan gracias a la amplitud de interconexión que ofrecen estas plataformas a los individuos (Van Dijck, 2016; Reguillo, 2017). En estas redes los vínculos abren el camino a la interconexión con sujetos colectivos (organizaciones civiles), ideológicos (partidos políticos, grupos religiosos), mediáticos (periodistas, líderes de opinión, medios de comunicación) y como identifica Latour (2008), aquellos vínculos con objetos y sujetos no humanos. Así, estas redes no solo resultan de una ampliación de las ya existentes, sino que obligan a desarrollar 
procesos específicos para su mantenimiento y fortalecimiento en el escenario sociodigital (Quinn \& Papacharissi, 2018).

Finalmente, se encuentran las redes sociodigitales que son aquellas tramas que combinan las dos estructuras reticulares anteriores. Estas redes tienen un carácter más relacionado a las lógicas operativas de cada plataforma como los algoritmos y las interfaces, así como a los flujos de información que transitan permanentemente por los usuarios y sus conexiones. En este tercer tipo de redes se encuentran aquellas arquitecturas reticulares que se forman por el contenido; las redes de interacción-comunicación son un ejemplo de estos nuevos procesos de socialización. Estas redes se estructuran con los enlaces que se forman alrededor del contenido con el que interactúan dos o más usuarios; este proceso puede terminar en una relación operativa perdurable como el seguir / ser seguido en Twitter o mediante la amistad en Facebook. Esto es importante porque con su formalización bajo las lógicas prestablecidas en cada plataforma se pueden abrir caminos a nuevos flujos de información que refuercen su vínculo o amplíe los ya existentes.

De esta manera, en este último tipo de redes se encuentra el objeto de estudio de esta investigación. La red conformada alrededor del hashtag \#LGBT permite observar y analizar aquellos vínculos efímeros en un primer momento, que desarrollan los usuarios con el contenido y con otros usuarios que interactúan con dicho hashtag.

Aquí es donde la propuesta de cartografías conectivas adquiere sentido y materialidad. Este concepto pretende atender las redes que se forman en el espacio digital como resultado de la confluencia de las redes que el individuo desarrolla en espacios como Facebook y Twitter. La palabra cartografía alude al sentido territorial de Internet en tanto espacio o lugar de encuentro para la socialización; la red en este espacio no tiene fronteras físicas per se, sino que los únicos márgenes que genera están dados por los elementos que la constituyen: perfiles e interacciones, las cuales desde el paradigma de la red serían nodos y vínculos respectivamente. Estos dos elementos dinamizan permanentemente los bordes y la propia naturaleza de la estructura reticular que se articula en el mundo digital.

En este sentido, existe una relación dialéctica entre la red, los nodos y sus vínculos. La red es más que la suma de sus partes, mientras que los nodos y los vínculos no solo son productores de redes, sino también productos de estas cartografías conectivas.

Por su parte, la palabra conectiva descansa en la estructura tanto de Internet como de las plataformas que allí se instalan; son las interacciones entre los usuarios/nodos lo que otorga el sentido reticular y la propia vitalidad a la red entendida como mapa o cartografía. Además, la conectividad de estas redes se encuentra en los recursos tales como el hashtag, meme, video, imagen o link que en conjunto responden a parte de los repertorios de acción conectiva (Reguillo, 2017). En estos recursos existe una capacidad de trazabilidad y una 
potencialidad distributiva: permite rastrear a quienes lo han usado, compartido o comentado, y su propio uso puede alcanzar una magnitud multiescalar dentro de las plataformas donde fluye.

Entonces la noción de cartografías conectivas resulta un concepto útil que representa el resultado de la hibridación de redes, de nodos y vínculos en el nuevo contexto onlife. El concepto se pluraliza de manera tácita porque una red no es estática, aunque ya esté delimitada; su visualización y estructura puede variar derivado de los indicadores y de las posiciones estratégicas que adquieren los nodos que la constituyen de acuerdo con los indicadores del ARS.

Por tanto, una red, o cartografía conectiva, tiene una posibilidad amplia de visualización: existe una extensa posibilidad de representación a partir de sus indicadores, de sus vínculos y de sus nodos. Así, las cartografías conectivas permiten mapear, visualizar y entender cómo la noción de red se encuentra presente en este tipo de procesos onlife.

Una vez delineado este marco teórico y conceptual, es menester evidenciar de qué manera es posible aplicar el ARS para poder obtener, visualizar y analizar estas cartografías conectivas.

\section{Metodología}

El ARS tiene una larga tradición sociológica (Lozares, 1996; Watts, 2006; Wasserman \& Faust, 2013). Su uso en Internet empezó a la par de la propia web 1.0 (Rogers, 2018) y ha cobrado mayor intensidad derivado de la exponencial presentación y representación de los individuos en estas plataformas (Ricaurte \& Ramos-Vidal, 2015).

Dado que las redes en el espacio virtual pueden variar en cuanto a nodos y vínculos, los softwares para trabajar mediante la minería de datos se han vuelto cada vez más importantes (Gunter, 2014; Olmedo Neri, 2019) y han implicado procesos paralelos de capacitación para trabajar con grandes cantidades de información. Entre los softwares para la obtención y visualización de estas redes se encuentra NodeXL, Virtual Observatory of Social Online Networks (Voson), R, Gephi, Pajek, Structure, Netdraw, entre otros (Rodríguez, 2005). Para este estudio se utilizaron los softwares NodeXL para la extracción de información y Gephi para la visualización de las cartografías conectivas.

Entonces, se puede observar un conjunto de implicaciones sobre el ARS que van desde los periodos de recolección de información, pasando por los procesos de sistematización y la selección de los propios indicadores para analizar una red.

En este trabajo se ha realizado una recolección de datos a través de las interacciones desarrolladas por usuarios alrededor de varias versiones del hashtag \#LGBT, ${ }^{1}$ las cuales tuvieron un incremento de uso durante junio de

1 Dado que el movimiento LGBT+ aglutina diferentes comunidades sexogenéricas y de identidades no heteronormadas existen varios hashtags que dan sentido a esta identidad colectiva. De allí que la inves- 
2020, mes donde confluyen diversas actividades culturales y de movilización LGBT en diferentes países. El uso del hashtag como variable de recolección deriva que esta etiqueta puede mostrar los usuarios y las formas en que interactuaron con dicha herramienta. Ya que los softwares tienen un alcance temporal de recolección corto se realizaron cinco procesos de recolección durante junio (cada 6 días). Con esto es posible adquirir la mayor cantidad de vínculos y reducir la posibilidad de repetición de datos.

Así, el periodo de recolección no solo responde a un carácter de apropiación, sino también atiende la sincronía existente entre las acciones de movilización y complementación con las acciones en Internet. Ya en otros estudios (Olmedo, 2020, 2021) se ha identificado que conforme se acercan las fechas de mayor actividad, por ejemplo, con la marcha del orgullo, la interacción en redes tiende a crecer, lo que muestra la sinergia entre las acciones de visibilidad relevantes de los propios movimientos y su amplificación mediante las plataformas sociodigitales.

Dado que esta investigación pretende observar y analizar la red de interacción-comunicación generada durante ese periodo de tiempo, la única depuración que se hizo ${ }^{2}$ tuvo la finalidad de retirar aquellos nodos que poseían única y exclusivamente vínculos reflexivos, ${ }^{3}$ dado que los objetivos de esta aproximación están enfocados en la parte conectiva de la interacción. En este sentido, de la matriz de datos obtenida mediante NodeXL se filtraron aquellos vínculos donde el productor y el destino eran el mismo nodo y se eliminaron. La siguiente tabla muestra la diferencia de nodos antes y después del proceso de sistematización y depuración.

Tabla 1. Número de nodos antes y después de la limpieza de datos

\begin{tabular}{|l|l|}
\hline $\begin{array}{l}\text { Número de nodos en la red (incluyendo usuarios con } \\
\text { vínculos reflexivos) }\end{array}$ & $\begin{array}{l}\text { Número de nodos en la red (sin incluir los usuarios con } \\
\text { vínculos reflexivos) }\end{array}$ \\
\hline 23.743 & 20.512 \\
\hline
\end{tabular}

Fuente: Elaboración propia a partir de datos obtenidos en Gephi

Finalmente, y como parte del objetivo que guía la presente investigación, se han seleccionado tres indicadores dentro del ARS con los cuales es posible analizar estructuralmente esta cartografía conectiva, al mismo tiempo que permite identificar aquellos nodos que adquieren una posición estratégica en dicha red. En la siguiente tabla se muestran los indicadores y sus definiciones.

tigación recolectó información de los siguientes hashtags \#LGBT, \#LGBTT, LGBTTT, LGBTTTI, \#LGBTTTIQ y \#LGBTTTIQA, para agruparlas bajo una misma noción: \#LGBT.

2 A partir de la información que se puede extraer, es posible realizar análisis centrados y delimitados a la ubicación, la fecha de publicación, el idioma, o el contenido que se desarrolla junto al hashtag.

3 Los vínculos reflexivos son todos aquellos lazos en los que el origen y destino de este es un mismo nodo, es decir, no conecta con nadie más que consigo mismo. 
Tabla 2. Indicadores estructurales para la red

\begin{tabular}{|l|l|}
\hline Indicador & Definición \\
\hline Densidad de red & $\begin{array}{l}\text { "La densidad de un grafo es la relación entre el número de líneas existentes } \\
\text { dividido por el número de líneas posibles" (Paniagua, 2012, p. 37). }\end{array}$ \\
\hline Nivel de grado & $\begin{array}{l}\text { "El grado de un nodo es la suma de las líneas que tiene ese nodo, los vínculos } \\
\text { directos que tiene cada uno" (Paniagua, 2012, p. 37). Cuando la red es de tipo dirigida } \\
\text { se puede desglosar este indicador en grados de entrada y grados de salida. }\end{array}$ \\
\hline Grado de intermediación & $\begin{array}{l}\text { "Un actor es central si se encuentra entre otros actores justo en sus geodésicas, } \\
\text { lo que implica que para tener una gran centralidad de «intermediación» el } \\
\text { actor debe estar entre muchos de los actores a través de sus líneas geodésicas" } \\
\text { (Wasserman \& Faust, 2013, p. 210). }\end{array}$ \\
\hline
\end{tabular}

Fuente: Elaboración propia

A partir de estos indicadores se puede reconocer la cantidad de nodos, de vínculos y sobre todo la dinámica relacional (Pisani \& Piotet, 2009) de perfiles que adquieren una condición de hub (Barabási, 2011), es decir, nodos que por los vínculos que posee y la direccionalidad de los mismos, se configura como un elemento que puede incrementar la difusión de un contenido o restringirlo de acuerdo con sus intereses (Aguilar Gallegos, Martínez González \& Aguilar Ávila, 2017). De esta manera, el ARS adquiere sentido en la presente investigación y permite mapear este tipo de cartografías conectivas a partir del hashtag.

\section{Resultados}

La red obtenida alrededor del hashtag \#LGBT durante junio de 2020 está constituida por 20.512 nodos y 25.184 enlaces. La distribución de los vínculos depende de la interacción de los usuarios con el contenido y el hashtag, por un lado, y el impacto que derivó de ello en sus redes sociales y digitales, por otro.

El tipo de red es dirigida, lo que indica que se conoce el origen y destino de los vínculos, por lo que este atributo será relevante para conocer el flujo de información dentro de esta estructura reticular. De acuerdo con el primer indicador establecido, es posible decir que la red tiene una densidad muy baja, prácticamente cero, lo cual significa que la cohesión existente entre los nodos es mínima. Lo anterior no significa que la cohesión no sea importante, sino que detrás de esa aparente desvinculación existe otra lógica, otra racionalidad que se fundamenta tanto en el objetivo del hashtag como en la propia plataforma donde se difunde: la viralización y su potencial mutación en un Tema Tendencia (Trending Topic, TT). La idea de llegar a la mayor cantidad de personas y con ello incrementar la visibilidad del hashtag y quienes lo utilizan son dos elementos que intervienen en la cohesión de los usuarios y la red.

Además, hay que tener presente que es una red internacional donde confluyen diferentes idiomas que concuerdan al usar LGBT como signo (como el inglés, el portugués y el español), por lo que la posibilidad de conexión después de esta actividad es posible, pero no necesaria. La cartografía conectiva que 
encarna este proceso de interacción y comunicación se muestra en la siguiente figura.

Figura 1. Red \#LGBT, junio 2020

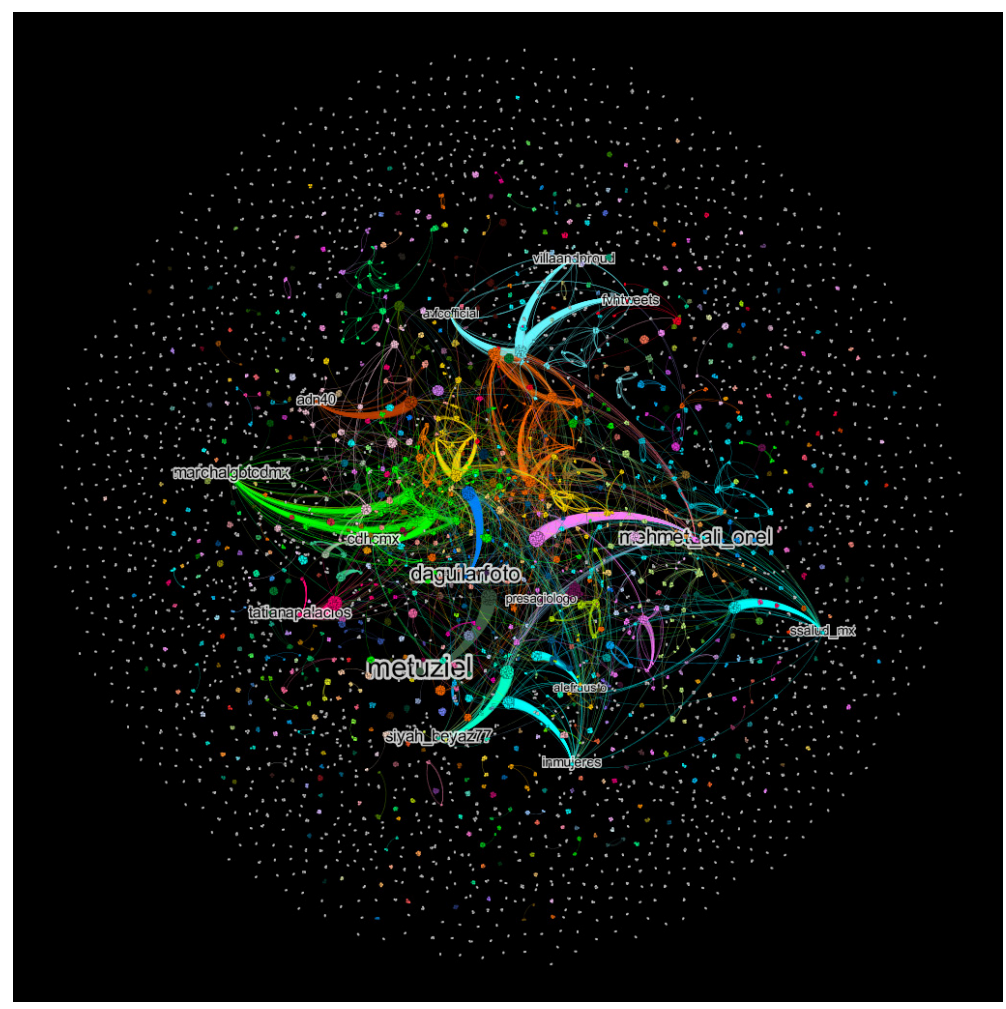

Fuente: Elaboración propia con datos de NodeXL y Gephi

Para esta cartografía conectiva se ha empleado el algoritmo de modularidad, con el cual se identifican las comunidades internas que integran esta red; cada color representa una comunidad o en términos de redes, un subgrafo.

El algoritmo de modularidad no solo ayuda a visualizar las comunidades, sino a entender parte de esta cartografía: este recurso permite identificar cómo los nodos se distribuyen dentro de la red y cuántas comunidades/subgrafos se han formado. Derivado de esto, se reconocieron 2399 comunidades. La siguiente gráfica muestra la distribución de esas comunidades mediante su tamaño. 
Gráfica 1. Distribución de comunidades por tamaño

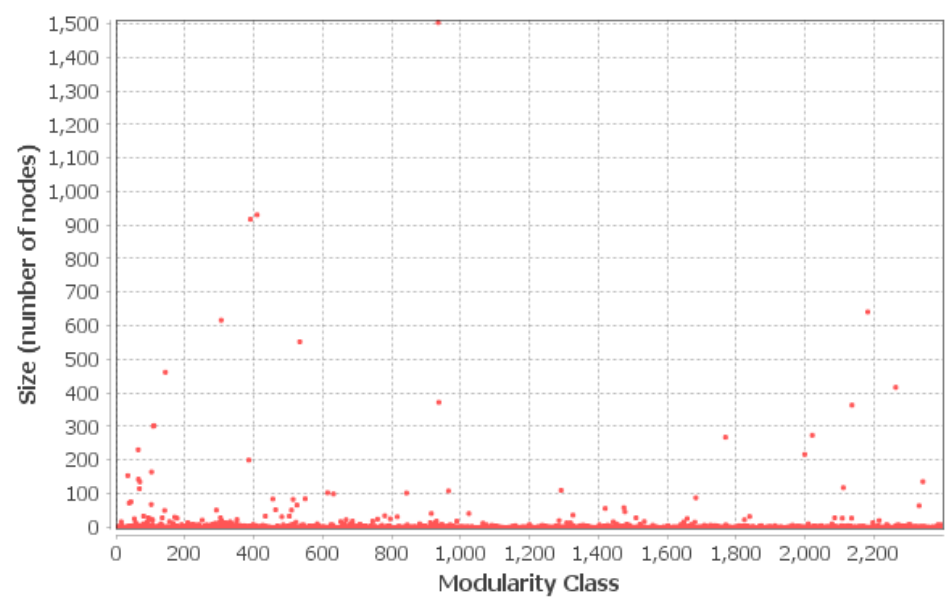

Fuente: De Blondel, Guillaume, Lambiotte y Lefebvre (2008); Lambiotte, Delvenne y Barahona (2009)

Con esta gráfica es posible reconocer que en esta red predominan las comunidades pequeñas y que muy pocas sobrepasan la frontera de 100 nodos por grupo. Así, es factible entender que en la Figura 1 existe un centro donde se sitúan las comunidades con mayor número de conexiones, mientras que en la periferia se evidencian estas comunidades pequeñas que interactúan entre sí.

Por otro lado, es importante mencionar la lógica de los nombres de usuarios que allí aparecen: esos perfiles son los que dentro de la red poseen la mayor cantidad de enlaces, es decir, se posicionan alrededor por la cantidad y no necesariamente por la direccionalidad de sus vínculos. La siguiente tabla identifica los tres nodos con más cantidad de vínculos.

Tabla 4. Nodos con mayor nivel de grado

\begin{tabular}{|l|l|l|c|c|c|c|}
\hline \multicolumn{1}{|c|}{ Nodo } & \multicolumn{1}{|c|}{ País $^{4}$} & \multicolumn{1}{c|}{ Rol $^{5}$} & $\begin{array}{c}\text { Grado de } \\
\text { entrada }\end{array}$ & $\begin{array}{c}\text { Grado de } \\
\text { salida }\end{array}$ & $\begin{array}{c}\text { Nivel de } \\
\text { grado }\end{array}$ & $\begin{array}{c}\text { Grado de } \\
\text { intermediación }\end{array}$ \\
\hline metuziel & Sin Dato & Miembro LGBT+ & 1,508 & 1 & 1.509 & 0 \\
\hline mehmet_ali_onel & Turquía & Periodista & 972 & 3 & 975 & 0 \\
\hline tatianapalacios & México & Aliada LGBT+ & 583 & 3 & 586 & 0 \\
\hline
\end{tabular}

Fuente: Elaboración propia con datos de Gephi

4 Dato obtenido a partir de la información pública de cada perfil.

5 Refiere a su profesión (abogado, investigador, organización) o su relación con la comunidad LGBT+ (miembro, activista, aliado, por ejemplo). 
Como se observa, estos nodos se ostentan en la red con el mayor número de vínculos, sin embargo, es importante dos particularidades: la primera es que los tres nodos poseen muy pocos vínculos de salida, mientras que el segundo factor es que ninguno de ellos posee grado de intermediación.

La relevancia de conocer la direccionalidad de los vínculos recae en que permite entender el impacto del contenido o la actividad que genera/comparte en su cuenta. Cuando una cuenta es etiquetada en un tuit o en un comentario se genera un vínculo de entrada, mientras que cuando una cuenta publica o comenta algún contenido se crea un vínculo de salida (Olmedo Neri, 2019). Con esta diferenciación en la dirección de los vínculos se infiere que estos usuarios publicaron muy poco contenido relacionado al hashtag \#LGBT, en comparación al impacto que tuvieron en sus redes. Por ello resulta relevante saber el contenido de sus publicaciones para conocer de cierta manera la razón que les generó esta capacidad de alcance.

Las siguientes figuras evidencian el contenido publicado desde estos perfiles.

Figura 2. Tuit de @metuziel

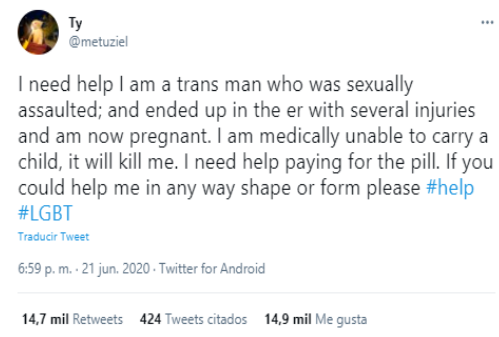

Fuente: Ty (2020)
Figura 3. Tuit de @mehmetali_Onel

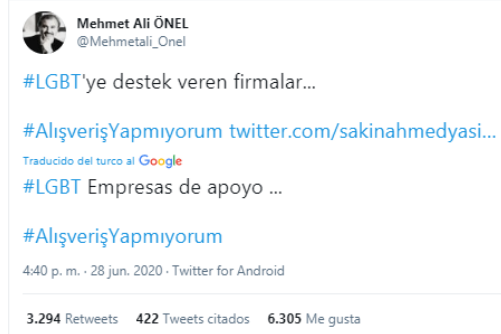

Fuente: Ali Önel (2020)

Figura 4. Tuit de @tatianapalacios

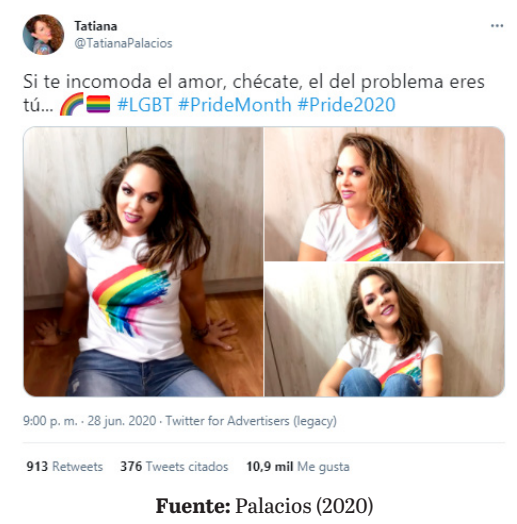


Con esto es posible reconocer tres contextos que evidencian la pluralidad no solo del contenido, sino también los retos que este movimiento social enfrenta en su vida cotidiana. Mientras que en el primer caso se manifiesta una situación derivada de la agresión física, psicológica y sexual a una persona integrante de la comunidad Trans, en la segunda figura se difunde un producto informativo que da visibilidad a las empresas aliadas y en la tercera es una apuesta a una de las demandas y recursos discursivos del propio movimiento.

Referente al nivel de grado, es necesario esbozar que una cantidad significativa de vínculos no necesariamente corresponde a una posición estratégica en el flujo de información. En términos específicos esto aplica a solo poseer grados de entrada o de salida. Para dar mayor sustento a esto, en la siguiente tabla se muestran los tres nodos con mayor grado de salida dentro de la cartografía conectiva alrededor del hashtag \#LGBT.

Tabla 5. Nodos con mayor número de vínculos de salida

\begin{tabular}{|l|l|l|l|l|l|l|}
\hline \multicolumn{1}{|c|}{ Nodo } & \multicolumn{1}{|c|}{ País } & \multicolumn{1}{|c|}{ Rol } & $\begin{array}{c}\text { Grado de } \\
\text { entrada }\end{array}$ & $\begin{array}{c}\text { Grado de } \\
\text { salida }\end{array}$ & $\begin{array}{c}\text { Nivel de } \\
\text { grado }\end{array}$ & $\begin{array}{c}\text { Grado de } \\
\text { intermediación }\end{array}$ \\
\hline aledlloydrees & Inglaterra & Blogger LGBT+ & 2 & 41 & 43 & 84 \\
\hline alesitaperez & México & Sin dato & 0 & 28 & 58 & 0 \\
\hline captasacl & México & Organización LGBT+ & 1 & 24 & 25 & 92 \\
\hline
\end{tabular}

Fuente: Elaboración propia a partir de datos de Gephi

A partir de los datos obtenidos se identifica que las posiciones de los nodos no son estáticas, sino que responden al indicador o al interés de la investigación, de allí que incluso delimitada, la red se modifica de manera interna, lo que evidencia una pluralidad de redes dentro de la red. Con estos resultados se muestra que: 1. Los indicadores poseen una autonomía, y 2. Una cantidad de vínculos, sean de entrada o salida, no necesariamente corresponden a una posición estratégica dentro del flujo de información.

Para poder materializar esta diferencia entre indicadores, en la siguiente tabla se han identificado los tres nodos que se posicionan estratégicamente en el flujo de información que recorre los vínculos y los nodos de esta red de redes.

Tabla 6. Nodos con mayor grado de intermediación

\begin{tabular}{|l|l|l|l|l|l|l|}
\hline \multicolumn{1}{|c|}{ Nodo } & \multicolumn{1}{|c|}{ País } & \multicolumn{1}{|c|}{ Rol } & $\begin{array}{c}\text { Grado de } \\
\text { entrada }\end{array}$ & $\begin{array}{c}\text { Grado de } \\
\text { salida }\end{array}$ & $\begin{array}{c}\text { Nivel de } \\
\text { grado }\end{array}$ & $\begin{array}{c}\text { Grado de } \\
\text { intermediación }\end{array}$ \\
\hline gabrielgtzg & México & Miembro LGBT+ & 7 & 9 & 16 & 5.863 \\
\hline marchalgbtcdmx & México & $\begin{array}{l}\text { Generador de } \\
\text { contenido LGBT+ }\end{array}$ & 188 & 7 & 195 & 5.727 \\
\hline antoniomedina41 & México & $\begin{array}{l}\text { Activista/ } \\
\text { Investigador } \\
\text { LGBT+ }\end{array}$ & 31 & 18 & 49 & 4.807 \\
\hline
\end{tabular}

Fuente: Elaboración propia a partir de datos de Gephi 
Una vez más, los usuarios cambian de acuerdo con el indicador; una nueva red emerge de la anterior y con ellos nuevas posiciones. En este caso, el grado de intermediación dota de un atributo de difusión al usuario dentro del flujo de información, volviéndolo un hub (Barabási, 2011), es decir, un usuario con la capacidad de incrementar o restringir el alcance de un contenido dentro de la red.

Resulta destacable que, para este indicador, los tres perfiles están ubicados en México y tienen una relación directa con la comunidad LGBT+, ya sea como miembro, activista o generador de contenido. En este sentido, el grado de intermediación evidencia la cantidad de caminos en los que estos nodos participan para conectar a otros dos perfiles dentro de la estructura, por lo que, en una red con poca cohesión interna, estos nodos adquieren un papel relevante en la difusión y alcance de la información.

\section{Discusión}

A partir de los resultados obtenidos es posible abonar al estudio de las formas en que el movimiento LGBT utiliza Internet para visibilizar su presencia y las demandas que motivan su horizonte de posibilidades.

Dentro de la discusión contemporánea alrededor de este objeto de estudio (Jiménez Zarate, 2017; Olmedo Neri, 2020, 2021; Peirone, 2012; Sierra Caballero \& Sola-Morales, 2020; Sola-Morales \& Sabariego Gómez, 2020) resulta necesario reconocer que estas estrategias de acción y comunicación adquieren un carácter emergente que requieren una visión y análisis interdisciplinario alrededor, entre otros temas, del carácter reticular de la acción conectiva (Reguillo, 2017; Rovira, 2017). El uso de las plataformas sociodigitales no solo atiende la fascinación técnica devenida en presión social por "estar en línea” (García Calderón \& Olmedo Neri, 2019), por el contrario, se desarrollan nuevos procesos de socialización que muestran un carácter híbrido propio del contexto contemporáneo: las redes se manifiestan en la red como resultado de la interacción social y de la incorporación de la tecnología en la vida cotidiana.

Así, la red formada alrededor del conjunto de hashtags \#LGBT es una fotografía que conjuga la esencia reticular de la organización del movimiento LGBT+ a escala internacional y la estructura de Twitter e Internet. Esto muestra que el movimiento LGBT+, el cual remonta sus primeras acciones organizativas en la lucha iniciada en 1969 en StoneWall (Martel, 2013), también ha reconocido en el espacio digital un campo fértil para la intervención eincidencia informativa, simbólica y cultural, por lo que el estudio alrededor de las funciones que le da en la actualidad abre un campo para la investigación social.

La (inter)conexión desarrollada mediante los lazos muestra que el movimiento LGBT+ posee una reticularidad estructural; sus integrantes actúan de manera autónoma $\mathrm{y}$, sin embargo, todas las acciones dispersas contribuyen en conjunto a alcanzar sus metas al largo plazo. El uso de estas 
plataformas también tiene un carácter de profunda intervención: sus luchas encuentran eco dentro de las redes de la red. Con esta posibilidad comunicativa se eliminan los intermediarios, se articulan discursos alternativos a los estigmas históricamente construidos sobre la comunidad LGBT+ y es posible incrementar la visibilidad de su agenda dentro de la esfera pública construida en plataformas como Twitter. Con este primer acercamiento se observa cómo la reivindicación cultural, social, política y comunicativa adquiere un papel preponderante dentro de este movimiento social.

La diversidad de comunidades dentro de la red muestra que existe un trabajo local por mantener y reforzar los vínculos, aunque eso no demerita que existan vinculaciones a otras comunidades más grandes, ni tampoco significa que solo en un país exista este proceso de hibridación y convergencia. Por el contrario, este primer acercamiento permite ver que las comunidades internacionales están empezando a utilizar y consolidar el uso de estas plataformas para visibilizar sus demandas y luchas. Esto no es más que la construcción de repertorios de acción conectiva a partir de la apropiación social de la tecnología.

A nivel estructural, la posición estratégica de un nodo es variable de acuerdo con el indicador o el elemento a estudiar; de allí que su caracterización a través de su direccionalidad se convierte en un recurso útil para entender las dinámicas internas y los flujos informativos de estas cartografías conectivas. Además, cabe mencionar que una mayor cantidad de vínculos no necesariamente significa que el nodo publique o interactúe de manera constante, por el contrario, detrás de esos vínculos existen procesos de socialización que tienen fundamento en la biografía del individuo y su participación tanto en el movimiento como en la esfera pública. Por ello, futuras investigaciones pueden abrir camino a estudios que evidencien el fundamento interactivo, socializador o de poder comunicativo que sostiene dicho enlace y su capacidad amplificadora en las cartografías conectivas.

De los indicadores analizados destaca el caso del grado de intermediación dado que su construcción en el ámbito digital responde a un carácter más amplio que el mero contenido: el rol y la función que desempeña dentro de la comunidad y de la red formada. Es decir, un nodo solo tendrá una posición estratégica en el flujo de información en la medida que:1. Publique contenido de manera constante (y que sea de interés y calidad), 2. Que posea un reconocimiento ante los demás nodos dentro del escenario sociodigital y 3. Que dicho reconocimiento/prestigio sea legitimado por los propios vínculos que posee en sus tres tipos de redes. Ser reconocido o identificado como un actor/nodo relevante para una comunidad se convierte en un atributo que le da sentido y le otorga confianza entre los que integran el mapa conectivo, por lo que el rol que cumpla dentro de la red y en la comunidad en general, tendrá un peso relevante.

A nivel de interacción, las tres narrativas que atrajeron/desarrollaron una mayor cantidad de vínculos muestran los avances, la visibilidad y también los retos que todavía se manifiestan en contra de las comunidades que integran la 
población LGBT+ en el mundo. Sin importar la ubicación geográfica, queda claro que el movimiento debe continuar con su avance paulatino en el ámbito social, cultural, económico, político y comunicativo para materializar su horizonte de acción.

Finalmente, en cuanto a las comunidades, y de acuerdo con los perfiles que van posicionándose dentro de cada indicador, existe una presencia relevante de miembros del movimiento LGBT+ mexicano, dado que ostentaron seis de los nueve lugares más relevantes dentro del mapa conectivo. Esto resulta relevante dado que en ese movimiento comienza a visibilizarse el uso y la investigación alrededor de esta convergencia de acciones e interacciones dentro de Internet; sean utilizadas estas plataformas para difundir demandas como el matrimonio igualitario (Olmedo Neri, 2020), para la construcción de redes en y entre organizaciones de este movimiento (Ciszek, 2017; Enguix Grau, 2016), con la finalidad de incrementar la visibilidad y organización emergente entre las juventudes LGBT+ (Garay Cruz, 2018,) o para analizar el consumo cultural que se amplía en este mundo virtual (Hernández Muñoz, 2018), queda claro que este proceso se ha materializado de un campo fértil y relativamente nuevo que requiere atención desde las ciencias sociales, particularmente desde la comunicación.

\section{Conclusiones}

Este trabajo es un primer acercamiento a las cartografías conectivas que desarrolla el movimiento LGBT+ a escala internacional. El objetivo de abordar esas redes de manera estructural y a partir del contenido que allí generó más interacción trajo como resultado una muestra parcial de la realidad compleja que la comunidad vive en su cotidianidad.

A partir de retomar la noción de red como paradigma, resulta factible identificar, caracterizar y analizar la forma en que las redes sociales se crean o replican en el contexto híbrido, convergente e hipercomunicado. Los resultados muestran que esta red en realidad está conformada por redes regionales y locales que comparten rasgos culturales y lingüísticos, así como retos que enfrentar.

Desde el punto de vista estructural y analítico, la red tiene una relación dialéctica con sus partes constituyentes; los nodos y los vínculos no solo crean la red, sino que también son producto de esta arquitectura reticular. Estos dos elementos son partes que reflejan los procesos de interacción social a través del contenido, en este caso el hashtag. Por tanto, resulta importante recalcar que la red es más que la suma de sus partes y que mantiene con ellas la dinámica relacional que fundamenta la lógica digital en Internet. Además, es importante reconocer que con cada indicador los nodos "relevantes" cambian su posición, mostrando que incluso dentro de una red ya delimitada, esta encierra en sí misma una pluralidad de redes. 
La propuesta y apuesta en el concepto de cartografías conectivas descansa en la idea de no solo analizar las estructuras reticulares, sino en términos generales pensar y actuar en red. Esta metáfora, convertida hoy en eje rector de varios marcos teóricos y perspectivas de análisis, permite redimensionar estudios, abrir campos interdisciplinarios de conocimiento y proponer nuevas formas de abordar los movimientos sociales contemporáneos. Así, el concepto de cartografías conectivas se vuelve factible para reconocer las redes, la socialidad y el contenido que los movimientos sociales contemporáneos articulan en Internet; identificar a los usuarios a partir de su intervención en el espacio público, por el impacto de su contenido o por el papel que desarrollan al interior de su movimiento/comunidad implica reconocerles una posición dentro de la arquitectura reticular, la cual está fundada en el carácter comunicativo de su interacción. Por tanto, las posiciones de los nodos reflejan tanto la esencia comunicativa de su respectivo usuario como el papel dentro del movimiento y su reconocimiento por parte de la comunidad.

En otras palabras, conocer la posición de los nodos contribuye a entender las relaciones de poder, el activismo y la relevancia de quien crea y difunde contenido de interés para una comunidad que poco a poco se consolida en el imaginario social del siglo XXI.

Finalmente, con este primer acercamiento se ha visto que un movimiento de gran alcance histórico, político, cultural e identitario como el LGBT+ ya evidencia procesos de apropiación tecnológica con los cuales se materializan sus repertorios de comunic-acción. Esta forma de entender y analizar la interacción que se desarrolla en Internet, permite ampliar no solo el horizonte de posibilidades de sus propios integrantes, sino también mostrar un cambio sutil, pero de gran alcance que se está llevando a cabo dentro de la sociedad global contemporánea.

\section{Referencias bibliográficas}

Acosta, M. (2020). Diversidad sexual y espacio público digital. La dinámica comunicacional de los/las usuarios/as del hashtag \#LoveIsLove en Twitter. Hologramática, 2(33), 23-50.

Acosta, M., \& Lassi, A. (2020). La conversación digital durante la Huelga Internacional de Mujeres. Revista Arbitrada Interdisciplinaria Koinonia, V(9), 86-109.

Aguilar Gallegos, N., Martínez González, E. G., \& Aguilar Ávila, J. (2017). Análisis de Redes Sociales: Conceptos clave y cálculo de indicadores. Ciudad de México: UACh-CIESTAAM.

Ali Önel, Mehmet (mehmetali_onel) (28 de junio de 2020). \#LGBT'ye destek veren firmalar... \#AlışverişYapmıyorum https://twitter.com/sakinahmedyasin/status/1277206088723685376/video/1 \#LGBT Empresas de apoyo ...\#AlışverişYapmıyorum [Tuit]. Recuperado de https://twitter.com/Mehmetali_Onel/status/1277356239811489792

Barabási, A.-L. (2011). Introduction and Keynote to A Networked Self. En Z. Papacharissi, A Networked Self (p. 1-14). Nueva York: Routledge.

Bauman, Z., y Leoncini, T. (2018). Generación líquida. Ciudad de México: Paidós. 
Candón-Mena, J. (2019). Movimientos conectados: Abordajes tecnopolíticos. IC-Revista Científica de Información y Comunicación, 23-35.

Castells, M. (2010). Comunicación y Poder. Madrid: Alianza Editorial.

Castells, M. (2015). Redes de indignación y esperanza. Madrid: Alianza Editorial.

Ciszek, E. (2017). Todo mejora en el ambiente: An analysis of digital LGBT activism in Mexico. Journal of Communiation Inquiry, 41(4), 313-330.

Crovi Druetta, D. (2012). Apropiación: una aproximación conceptual. En M. Portillo Sánchez, \& I. Cornejo Portugal, ¿̇Cómunicación posmasiva? (p. 149-162). Ciudad de México: Universidad Iberoamericana.

D Blondel, Vincent; Guillaume, Jean-Loup; Lambiotte Renaud, \& Lefebvre, Etienne (2008). Fast unfolding of communities in large networks. Journal of Statistical Mechanics: Theory and Experiment (10). P1ooo.

Diodato, R. (2011). Estética de lo virtual. Ciudad de México: Universidad Iberoamericana.

Enguix Grau, B. (2016). Activismo y Prácticas Digitales en la Construcción de una esfera LGBT en España. Dados. Revista de Ciências Sociais, 59(3), 755-787. doi: https://doi. org/10.1590/00115258201691

Flores-Márquez, D. (2017). Movimientos sociales e Internet en México. En G. Pleyers, y M. Garza Zepeda (Coords.), Mexico en Movimientos. Resistencias y alternativas (p. 119-126). Ciudad de México: UACJ-MA Porrúa.

Floridi, L. (2015). The Onlife Manifesto. Being Human in a Hyperconnected Era. Nueva York: Routledge.

Fuchs, C. (2015). Culture and economy in the age of social media. Nueva York: Routledge.

Garay Cruz, L. M. (2018). Colectivos de diversidad sexual, redes sociodigitales y ciberactivismo como escenarios de visibilidad. En J. Candón Mena (Ed.), Actas del II Congreso Internacional Move.Net sobre Movimientos Sociales y TIC, celebrado del 25 al 27 de octubre de 2017 (p. 92-108). Sevilla: Compoliticas.

García Calderón, C., \& Olmedo Neri, R. A. (2019). El nuevo opio del pueblo: apuntes desde la Economía Política de la Comunicación para (des)entender la esfera digital. Iberoamérica Social, 7(XII), 84-96. https://n9.cl/h83nt

Gunter, B. (2014). Los procedimientos de las investigaciones cuantitativas. En K. B. Jensen, La comunicación y los medios (p. 379-424). Ciudad de México: Fondo de Cultura Económica.

Hernández Muñoz, M. (2018). Webseries y cultura digital en la comunidad LGBT en México. El caso de la webserie “Con Lugar”. En J. Cadena-Roa, M. Aguilar Robledo, \& D. E. Vázquez Salguero (Coords.), Las ciencias sociales y la agenda nacional. Reflexiones y propuestas desde las Ciencias Sociales (p. 1111-1130). Ciudad de México: Comecso.

Jiménez Zarate, C. A. (2017). Redvolución. Ciudad de México: Ld Books.

Kadushin, C. (2013). Comprender las redes sociales. Teorías, conceptos y hallazgos. Madrid: Centro de Investigaciones Sociológicas.

Lambiotte, R.; Delvenne, J. C; Barahona, M. (2009) Laplacian Dynamics and Multiscale Modular Structure in Networks.

Latour, B. (2008). Reensamblar lo social: una introducción a la teoría del actor-red. Buenos Aires: Manantial.

Lozares, C. (1996). La teoría de redes sociales. Papers (48), 103-126.

Lozares, C. (2005). Bases socio-metodológicas para el Análisis de Redes Sociales, ARS. Empiria. Revista de Metodología de las Ciencias Sociales (10), 9-35.

Luna, M. (2004). Redes sociales. Revista Mexicana de Sociología, LXVI(Número Especial), 59-75. 
Martínez Carmona, C. A., González Villarreal, R. J., Mogrovejo Aquise, M. N., \& Sandoval Rebollo, E. M. (2020). Movimientos LGBT en México. En G. Olivier (Coord.), Estado del conocimiento de los movimientos sociales en México (p. 281-341). Ciudad de México: SEPUPN.

Martel, F. (2013). Global Gay. Cómo la revolución gay está cambiando el mundo. Madrid: Taurus.

Mattoni, A., \& Treré, E. (2014). Media practices, mediation processes and mediatization in the study of social movements. Communication Theory, 24(3), 252-271.

Meneses Rocha, M. E. (2015a). Ciberutopías. Ciudad de México: Tecnológico de Monterrey-Porrúa.

Meneses Rocha, M. E. (2015b). Redes sociales virtuales: potencial democratizador y herramientas de vigilancia. En R. Winocur Iparraguirre, \& J. A. Sánchez Martínez, Redes sociodigitales en México (pp. 40-61). Ciudad de México: Fondo de Cultura Económica / Conaculta.

Olmedo Neri, R. A. (2019). \#AmorEsAmor como constructor de redes digitales en el movimiento LGBTTTIQA en México. Virtualis, 10(19), 109-133. https://www.revistavirtualis. $\mathrm{mx} / \mathrm{index} . \mathrm{php} /$ virtualis/article/view/301

Olmedo Neri, R. A. (2020). La comunicación emergente en México (2009-2016): las organizaciones civiles en la lucha por el matrimonio igualitario. (Tesis de Maestría) Ciudad de México: UNAM. Obtenido de https://bit.ly/3eU6jDs

Olmedo Neri, R. A. (2021). Luchar y comunicar en el siglo XXI. Mitos y aproximaciones desde \#LiberaTuOrgullo en México. En J. Hidalgo Toledo, M. Echeverría Victoria, M. Sánchez Maldonado, I. Lay Arellano, F. Acevez González, J. Esteinou Madrid, . . . I. Corduneanu (Coords.), Dimensiones de lo público y lo político en la segunda alternancia democrática. Socialización, participación alternativa y discurso político (p. 327-353). Aveiro: Ria Editorial.

Palacios, Tatiana (tatianapalacios) (28 de junio de 2020). Si te incomoda el amor, chécate, el del problema eres tú... \#LGBT \#PrideMonth \#Pride2020. [Tuit]. Recuperado de https:// twitter.com/tatianapalacios/status/1277421530159382529

Paniagua López, J. A. (2012). Curso de análisis de redes sociales. Metodología y estudios de caso. Granada: Universidad de Granada.

Peirone, F. (2012). Mundo extenso. Ensayo sobre la mutación política global. Buenos Aires: FCE.

Pedraza, C. I., y Rodríguez Cano, C. A. (2019). Resistencias sumergidas. Cartografías de la tecnopolítica feminista en México. Teknokultura. Revista de Cultura Digital y Movimientos Sociales, 197-212.

Pisani, F., \& Piotet, D. (2009). La alquimia de las multitudes. Barcelona: Paidós.

Quinn, K., \& Papacharissi, Z. (2018). Our Networked selves: Personal connection and relational maintenance in social media use. En J. Burguess, A. Marwick, \& T. Poell (Eds.), The SAGE Handbook of Social Media (p. 353-371). Londres: SAGE.

Reguillo, R. (2013). Culturas juveniles. Formas políticas del desencanto. Buenos Aires: Siglo XXI.

Reguillo, R. (2017). Paisajes Insurrectos. Guadalajara: Iteso.

Rheingold, H. (2004). Multitudes inteligentes. Barcelona: Gedisa.

Ricaurte, P., \& Ramos-Vidal, I. (2015). Investigación en redes sociales digitales: consideraciones metodológicas desde el paradigma estructural. Revista Virtualis, 165-194. 
Rodríguez, J. A. (2005). Análisis estructural y de redes. Madrid: Centro de Investigaciones Sociológicas.

Rodríguez Cano, C. A. (2020). La expansión de lo público. Indagaciones tecnopolíticas de la sociedad hiperconectada. Ciudad de México: UAM / Unidad Cuajimalpa.

Rogers, R. (2018). Digital methods for across-platform analysis. En J. Burgess, A. Marwick, \& T. Poell (Eds.), The SAGE Handbook of Social Media (p. 91-110). Londres: SAGE.

Rovira Sancho, G. (2012). Movimientos sociales y comunicación: la red como paradigma. Análisis, 91-104.

Rovira, G. (2017). Activismo en red y multitudes conectadas. Ciudad de México: Icaria.

Scolari, C. (2015). Ecología de medios. Barcelona: Gedisa.

Sierra Caballero, F., \& Sola-Morales, S. (2020). Espacio público oposicional y ciberactivismo, una lectura materialista de la acción conectiva. Perspectivas de la Comunicación, 13(7), 7-41.

Sola-Morales, S., \& Sabariego Gómez, J. (2020). Tecnopolítica, recientes movimientos sociales globales e Internet. Una década de protestas ciudadanas. Teknokultura. Revista de Cultura Digital y Movimientos Sociales, 17(2), 195-208.

Treré, E. (2020). Activismo mediático híbrido. Bogotá: Friedrich Ebert Stiftung.

Ty (metuziel) (21 de junio de 2020). I need help I am a trans man who was sexually assaulted; and ended up in the er with several injuries and am now pregnant. I am medically unable to carry a child, it will kill me. I need help paying for the pill. If you could help me in any way shape or form please \#help \#LGBT. [Tuit]. Recuperado de https://twitter. com/metuziel/status/1274854518375829504

Van Dijck, J. (2016). La cultura de la conectividad: Una historia crítica de las redes sociales. Buenos Aires: Siglo XXI.

Wasserman, S., \& Faust, K. (2013). Análisis de redes sociales. Métodos y aplicaciones. Madrid: Centro de Investigaciones Sociológicas.

Watts, D. J. (2006). Seis grados de separación, La ciencia de las redes en la era del acceso. Barcelona: Paidós. 